\title{
Do Millennials differ in terms of survey participation?
}

\author{
Oriol J. Bosch \\ Melanie Revilla \\ Ezequiel Paura
}

RECSM Working Paper Number 58

July 2018 


\title{
Do Millennials differ in terms of survey participation?
}

Oriol J. Bosch, RECSM-Universitat Pompeu Fabra

Melanie Revilla, RECSM-Universitat Pompeu Fabra

Ezequiel Paura-Netquest

\begin{abstract}
Millennials have been the focus of quite some research because of their differences with older cohorts. Besides, young respondents have been considered as a hard target population for surveys. However, to our knowledge, no research has compared the levels and types of survey participation of the Millennials versus the older generations. Using a dataset of 1,570,301 panelists of an opt-in online panel in eight countries from Europe, Latin America and North America, we show that Millennials differ from older cohorts in terms of survey participation. Millennials show lower participation rates than older cohorts. Moreover, they present significantly higher proportions of surveys answered using smartphones. However, differences across cohorts in terms of break-offs and survey evaluation are mainly nonsignificant and/or nonmeaningful.
\end{abstract}

Keywords: Millennials, survey participation, break-off, smartphones, survey evaluation

\section{Introduction}

Strauss \& Howe (1991) define Millennials as the cohort of individuals born between 1982 and 2003. Millennials experienced similar events during their life, which differentiate them from other cohorts at several levels (Bowen and Chen McCain, 2015): in particular, Millennials are the first to have had, during their formative years, access to the Internet (Pew Research Center, 2014). Consequently, Millennials are the generation with the highest technology exposure (Hartman and McCambridge, 2011).

In the last years, Millennials have been the focus of quite some research for several reasons. On the one hand, Millennials are currently young adults, and thus, a very interesting target for marketers and brands. On the other hand, Millennials seems to present some specificities that make necessary to adapt the way to approach them. For 
instance, Millennials have been found more independent and self-sufficient than other generations (Williams and Page, 2011). Hence, their use of self-service technology, that allows to avoid human interaction, is higher (Mayock, 2014). In addition, being a Millennial is positively related with being loyal toward brands (Gehan and Yamamoto, 2017).

One of the main levels where Millennials seem to differ is their way to communicate. Indeed, Millennials' communicative skills have been found of lower quality than the ones of previous generations (Hartman and McCambridge, 2011). However, this cohort has a greater affinity for Computer-Mediated Communication tools (Myers and Sadaghiani, 2010). For instance, in the USA, a Pew Research Center (2014) shows that $89 \%$ have a profile in a Social Network Site (SNS), versus 73\% for Generation X (1961-1981), 45\% for younger Boomers (1956-1960) and 54\% for older Boomers (1946-1955). In addition, the same study shows that Millennials have the highest average number of Facebook friends (250, versus 200 for Generation X, 98 for younger Boomers and 50 for older Boomers) and that $55 \%$ have posted one or more selfie(s) to SNSs, versus $24 \%$ of Generation $X$ and $9 \%$ of Boomers. As a result, Millennials process website information five times faster than older generations (Kim and Ammeter, 2008). Besides, thanks to technology and globalization, it seems that there are less cross-national differences for Millennials than for other generations (Stein and Sanburn, 2013).

Do these differences between Millennials and the other generations also apply to their survey participation? Do these different ways of communicating affect also how they share information in surveys? Different studies found an impact of age on survey participation and the device used to complete the survey (e.g. Lugtig and Toepoel, 2016; Revilla et al., 2016). However, to the best of our knowledge, there is no study really comparing the levels and types of survey participation of the Millennials versus the older generations (Generation X and Boomers).

We try to start filling this gap, focusing on the participation within the frame of nonprobability online panels, which represent the majority of online research nowadays (Baker et al., 2010). In such panels, once a person has accepted to participate, he/she receives regularly survey invitations. Each time the panelist gets an invitation, different scenarios are possible: 1) The panelist does not start the survey at all; 2) The panelist starts the survey but he/she is excluded because his/her profile does not fit the population 
of interest ("screened-out") or some quotas are already full; 3) The panelist starts the survey but decides at some point (it can be any moment after the start) to abandon it (“break-off”); 4) The panelist starts the survey and reaches the survey's final question.

Furthermore, even when the panelist completes the survey until the end, he/she might not be satisfied with the experience he/she got while participating to this survey. This can be linked to different aspects, in particular survey characteristics (topic, length, layout, etc), the panelist's preferences, and/or the type of device (PC, tablet or smartphone) used to complete the survey. A negative experience while answering a survey may affect the willingness to participate in the future invitations.

Therefore, the goal of this paper is to examine within an opt-in online panel the differences between Millennials, Generation X and Boomers, in terms of: 1) participation rate, 2) break-off rate, 3) devices used to participate in the surveys and 4) survey evaluation.

\section{Hypotheses}

Young adults have been considered a hard target population for surveys. Proposing web as a mode of data collection has been seen as a way of increasing their participation (Lugtig and Toepoel, 2016), since the Internet penetration is higher for this cohort: in the United States, $97 \%$ of Millennials are Internet users versus $92 \%$ of Generation X, 83\% of younger Boomers and 76\% of older Boomers (Pew Research Center, 2014). However, we expect that Millennials participation rate will be overall lower than those of older cohorts.

In addition, respondents may abandon the survey for several reasons, especially because they start doing something else (and then do not think/want to come back to the survey to finish it) or because they do not like it. According to the Microsoft Consumer Insights report "Attention Spans" (Microsoft Canada, 2015), age is correlated with attention span and Millennials in Canada have a lower rate of high sustained attention (31\%, versus $34 \%$ for the $35-54$ years and $35 \%$ for the $54+$ ). This would be linked to the volume of media consumption, social media usage, multi-screening behavior, that would result in 
Millennials being less effective at filtering out distractions. Thus, we expect more breakoffs for Millennials.

Moreover, because $88 \%$ of the Millennials in the United States have a smartphone versus $77 \%$ of Generation X, 59\% of younger Boomers and $46 \%$ of older Boomers (Pew Research Center, 2014), we expect a higher proportion of Millennials to participate into the surveys through smartphones. Since previous research found higher break-off rates for mobile respondents (see e.g. Buskirk \& Andrus, 2014), this, in turn, could contribute to higher break-off rates for Millennials.

Furthermore, it could also lead to surveys being evaluated more negatively, since the visibility might not be as good on smartphones due to the smaller screens, and since completion times have been found to be longer for mobile respondents (see e.g. Andreadis, 2015; Mavletova \& Couper, 2013). Thus, overall, we expect that they will evaluate the surveys more negatively.

To sum up, we want to test the following hypotheses:

H1: The participation rate is lower for Millennials than for Generation X and Boomers.

H2: The break-off rate is higher for Millennials than for Generation X and Boomers.

H3: The proportion of smartphone respondents is higher for Millennials than for Generation X and Boomers.

H4: Millennials evaluate overall the surveys more negatively than Generation $\mathrm{X}$ and Boomers.

\section{Method and data}

\subsection{Data}

We test these four hypotheses in eight countries from different regions: Europe (Portugal, Spain), Latin/central America (Argentina, Brazil, Chile, Colombia, Mexico), and North America (United States). 
We use data from the Netquest online fieldwork company (www.netquest.com). Netquest started its first online panel in 2006 and at May $1^{\text {rst }} 2018$, the company counted around 2.3 million of active panelists over 26 countries. In this study, we focus on the 1,570,301 panelists born between 1943 and 2003 who were considered as active at May $1^{\text {rst }}, 2018$, in the eight countries just mentioned (largest panels). Panelists are considered active if they have completed at least one of the last 12 surveys received. If they do not answer any of the last 12 questions, the Netquest team sends them an e-mail asking if they want to continue being panelists. If they answer affirmatively, they become active panelists again. For each panelist, we consider all the surveys he/she has been invited to during one year ( $1^{\text {rst }}$ of May 2017- $1^{\text {rst }}$ of May 2018) in order to compute the participation and breakoff rates and the proportions of smartphone respondents. For the survey evaluation, only the surveys programmed by Netquest can be used, since this information is not available when the surveys are programmed directly by the clients (this reduces the total $\mathrm{N}$ from $1,570,301$ to 502,410$)$.

Netquest sends panelists survey invitations via email, using a list of individuals who have agreed to receive emails after answering a short satisfaction survey on a website belonging to one of the company's many collaborators. The email invitation does not provide any information about the survey itself (in particular, nothing about the length or the topic), so the decision of participating can be seen as a measure of panelists loyalty.

Overall, most surveys are about food/beverages, politics/society, or health (Revilla, 2017) and most surveys use quotas (95.2\% of the 186 surveys studied by Revilla, 2017; the most used quotas being gender and age). Typically, respondents are not allowed to skip questions or to go back, even if there are some exceptions. Most surveys are optimized for mobile devices, but not all, and a significant proportion of respondents use mobile devices to complete the surveys, even if there are differences across countries (Revilla et al., 2016)

Respondents who get to the end of a survey are asked to evaluate the survey using one to five stars. The survey evaluations, overall, are quite positive (median of 4.1 across the 186 surveys studied by Revilla, 2017).

For each survey completed, panelists are rewarded with points which can be exchanged for gifts. The number of points is calculated based on the estimated length of the 
questionnaire (not on the actual one) and the points are only received if a survey is completed until the end.

\subsection{Method/Analyses}

Using the data just mentioned, we compare Millennials to Generation $\mathrm{X}$ and Boomers within each country, for each of these four aspects:

- The (annual) participation rate for each panelist, defined as the number of surveys he/she started from $1^{\text {rst }}$ of May 2017 to $1^{\text {rst }}$ of May 2018, divided by the number of surveys to which he/she was invited during the same time frame. Then, we compute and report the average of these individual participation rates, for Millennials, Generation $\mathrm{X}$, and Boomers, per country.

- The (annual) break-off rate for each panelist, defined as the number of times he/she abandoned a survey divided by the number of times he/she started a survey from $1^{\text {rst }}$ of May 2017 to $1^{\text {rst }}$ of May 2018. Then, we compute and report the average of these individual break-off rates, for each age cohort and country.

- The (annual) rate of surveys answered with smartphones for each panelist, defined as the number of surveys he/she participated using a smartphone divided by the number of times he/she started a survey from $1^{\text {rst }}$ of May 2017 to $1^{\text {rst }}$ of May 2018. The device used to start the survey, measured using paradata about the user-agent-strings, is the one considered (i.e. if a respondent switch to another device later on, this is not taken into account). Then, we compute and report the average of these individual rates of surveys completed through smartphones, for each age cohort and country.

- The evaluation of the surveys, measured using the question that Netquest proposes at the end of each survey programmed by the company to its panelists, asking them: "finally, what do you think about the survey? Select one to five stars to indicate if you think that the survey was (1) very badly done to (5) very well done". We compute and report the proportion of panelists with an average across all surveys of four stars or more, for each age cohort and country. 
Within each country, we use two-sample T-tests to compare averages and Z-tests to compare proportions, always testing Millennials versus another cohort. Since we use a large sample, even small differences can be statistically significant. Hence, to analyze if differences are meaningful, we also compute and report the effect sizes (Cohen's h).

\section{Results}

\subsection{Participation rate}

The first indicator used to compare Millennials with other cohorts was the annual participation rate. Table 1 presents these average participation rates per country and cohort.

Table 1: Average participation rate (in \%)

\begin{tabular}{l|c|c|c|c|c|c}
\hline \multicolumn{1}{c|}{ Country } & $\mathbf{N}$ & Millennials & Generation X & Boomers & $\begin{array}{c}\text { Cohen's h } \\
\text { Millennials- } \\
\text { Generation X }\end{array}$ & $\begin{array}{c}\text { Cohen's h } \\
\text { Millennials- } \\
\text { Boomers }\end{array}$ \\
\hline Portugal & 33,403 & 64.0 & $73.2^{*}$ & $79.1^{*}$ & .20 & .34 \\
\hline Spain & 183,532 & 64.3 & $69.5^{*}$ & $79.0^{*}$ & .11 & .33 \\
\hline Argentina & 249,809 & 59.2 & $57.3^{*}$ & $56.5^{*}$ & .04 & .05 \\
\hline Brazil & 364,240 & 52.5 & $62.9^{*}$ & $69.2^{*}$ & .21 & .34 \\
\hline Chile & 226,841 & 55.6 & $62.3^{*}$ & $68.1^{*}$ & .14 & .26 \\
\hline Colombia & 157,733 & 55.2 & $60.9^{*}$ & $66.8^{*}$ & .12 & .24 \\
\hline Mexico & 254,644 & 53.9 & $67.1^{*}$ & $75.6^{*}$ & .27 & .46 \\
\hline US & 100,099 & 36.5 & $43.0^{*}$ & $47.8^{*}$ & .13 & .23 \\
\hline
\end{tabular}

Note: the stars in the columns "Generation X" and "Boomers" indicate a significant difference between Millennials and the respective cohort. $* \mathrm{p}<.01$

First, there are differences across countries: European countries have higher average participation rates across all generations. The low participation rate shown by the United States may be related with the fact that it is the younger panel of all presented, so the probability of having loyal panelists is lower. In addition, differences across countries are similar for all generations.

Furthermore, the average participation rate of Millennials is significantly lower than the one of other cohorts in all countries except Argentina. The differences go up to 21.7 percentage points in Mexico (between Boomers and Millennials). The effects sizes when 
comparing Millennials and Generation X are mostly small or very small (<.2). Compared to Boomers, effects sizes are small to medium (between .23 and .46). Hence, we find support for our first hypothesis: Millennials present significantly lower participation rates than older generations.

\subsection{Break-off rate}

Next, we compare the annual break-off rate. Table 2 presents the averages per cohort and country.

Table 2: Average break-off rates (in \%)

\begin{tabular}{l|c|c|c|c|c|c}
\hline \multicolumn{1}{c|}{ Country } & $\mathbf{N}$ & Millennials & Generation X & Boomers & $\begin{array}{c}\text { Cohen's h } \\
\text { Millennials- } \\
\text { Generation X }\end{array}$ & $\begin{array}{c}\text { Cohen's h } \\
\text { Millennials- } \\
\text { Boomers }\end{array}$ \\
\hline Portugal & 33,139 & 12.2 & $13.8^{*}$ & 11.4 & .05 & .02 \\
\hline Spain & 182,102 & 15.2 & 15.3 & $11.9^{*}$ & .00 & .10 \\
\hline Argentina & 231,123 & 16.7 & $20.2^{*}$ & $20.2^{*}$ & .09 & .09 \\
\hline Brazil & 358,485 & 18.4 & $19.0^{*}$ & $16.8^{*}$ & .02 & .04 \\
\hline Chile & 213,894 & 20.0 & $21.9^{*}$ & $19.3^{*}$ & .05 & .02 \\
\hline Colombia & 145,644 & 15.6 & $17.9^{*}$ & 16.4 & .06 & .02 \\
\hline Mexico & 250,377 & 15.4 & $16.6^{*}$ & 14.9 & .03 & .01 \\
\hline US & 99,510 & 9.5 & $15.2^{*}$ & $16.3^{*}$ & .17 & .20 \\
\hline
\end{tabular}

Note: the stars in the columns "Generation X" and "Boomers" indicate a significant difference between Millennials and the respective cohort. $* \mathrm{p}<.01$

Latin American countries present higher average break-off rates than Spain, Portugal and the United States. Besides, cross-country differences are similar across generations.

Moreover, Millennials have a significantly higher average break-off rate than Boomers for four countries. However, they have a lower average break-off rate than Generation X for all countries (significant at the $1 \%$ level, except for Spain). In addition, all effect sizes are very small (<.2). Hence, our second hypothesis receives very little support: Millennials have higher break-off rates than Boomers in only half of the countries, and Generation X performs worse than Millennials.

\subsection{Device of participation}

Moving to the device of participation. Table 3 presents the average proportion of surveys answered using smartphones, per country and cohort. 
Table 3: Average proportion of surveys answered using smartphones (in \%)

\begin{tabular}{l|c|c|c|c|c|c}
\hline \multicolumn{1}{c|}{ Country } & $\mathbf{N}$ & Millennials & Generation X & Boomers & $\begin{array}{c}\text { Cohen's h } \\
\text { Millennials- } \\
\text { Generation X }\end{array}$ & $\begin{array}{c}\text { Cohen's h } \\
\text { Millennials- } \\
\text { Boomers }\end{array}$ \\
\hline Portugal & 33,109 & 57.4 & $32.4^{*}$ & $14.6^{*}$ & .51 & .94 \\
\hline Spain & 181,980 & 63.8 & $53.9^{*}$ & $33.9^{*}$ & .20 & .61 \\
\hline Argentina & 230,084 & 76.7 & $63.0^{*}$ & $33.8^{*}$ & .30 & .89 \\
\hline Brazil & 357,925 & 74.4 & $60.3^{*}$ & $34.6^{*}$ & .30 & .82 \\
\hline Chile & 213,541 & 71.2 & $61.2^{*}$ & $40.9^{*}$ & .21 & .62 \\
\hline Colombia & 145,393 & 59.9 & $47.9^{*}$ & $29.7^{*}$ & .24 & .62 \\
\hline Mexico & 249,929 & 72.1 & $54.1^{*}$ & $34.0^{*}$ & .38 & .78 \\
\hline US & 99,020 & 78.8 & $63.5^{*}$ & $36.2^{*}$ & .34 & .89 \\
\hline
\end{tabular}

Note: the stars in the columns "Generation X" and "Boomers" indicate a significant difference between Millennials and the respective cohort. $* \mathrm{p}<.01$

Spain, Portugal and Colombia have, in general, lower average proportions of surveys answered using smartphones. Hence, European countries deviate from Latin American countries and the United States. However, differences are more pronounced for Millennials than for Boomers, which present quite similar percentages across countries, with the exception of Portugal.

Furthermore, Millennials present significantly higher average proportions of surveys answered using smartphones than both Generation $\mathrm{X}$ and Boomers. Concretely, the proportion of surveys answered using smartphones for Millennials is between 9.9 and 25.0 percentage points higher than for Generation $X$ and between 20.2 and 42.9 percentage points higher than for Boomers. Differences between Millennials and Generation $X$ present small to medium effects sizes, whereas the effect sizes for the differences with Boomers range from .61 (medium) in Spain to .94 (large) in Portugal. Hence, differences are much higher between Millennials and Boomers, but our third hypothesis is supported for both cohorts.

\subsection{Evaluation of the surveys}

Finally, Table 4 presents the proportions of panelists with an average evaluation of four stars or more, per country and cohort. 
Table 4: Proportion of respondents with an average rating of four or more stars

\begin{tabular}{l|c|c|c|c|c|c}
\hline \multicolumn{1}{c|}{ Country } & $\mathbf{N}$ & Millennials & Generation X & Boomers & $\begin{array}{c}\text { Cohen's h } \\
\text { Millennials- } \\
\text { Generation X }\end{array}$ & $\begin{array}{c}\text { Cohen's h } \\
\text { Millennials- } \\
\text { Boomers }\end{array}$ \\
\hline Portugal & 13,073 & 71.2 & $67.0^{*}$ & $66.9^{*}$ & .09 & .09 \\
\hline Spain & 132,596 & 57.7 & $58.5^{*}$ & 58.9 & .02 & .02 \\
\hline Argentina & 55,604 & 70.1 & $68.3^{*}$ & 68.5 & .04 & .03 \\
\hline Brazil & 83,909 & 83.0 & $78.6^{*}$ & $74.9^{*}$ & .11 & .20 \\
\hline Chile & 73,872 & 78.9 & 79.1 & $81.7^{*}$ & .00 & .07 \\
\hline Colombia & 42,783 & 83.7 & 83.2 & 84.4 & .01 & .02 \\
\hline Mexico & 86,094 & 83.5 & 83.2 & 81.8 & .01 & .04 \\
\hline US & 14,479 & 86.7 & $85.2^{*}$ & $82.0^{*}$ & .04 & .13 \\
\hline
\end{tabular}

Note: the stars in the columns "Generation X" and "Boomers" indicate a significant difference between Millennials and the respective cohort. * $\mathrm{p}<.01$

Table 4 shows mixed results. A significantly higher proportion of Millennials in Portugal, Argentina, Brazil and the United States evaluated surveys with higher punctuations than Generation X and/or Boomers, whereas in Spain and Chile results go in the opposite direction. Besides, Colombia and Mexico do not present any significant differences across cohorts. In addition, all differences present very small effect sizes (<.2). Therefore, there is no clear pattern across countries nor across cohorts. Thus, our last hypothesis is not supported.

\section{Discussion and conclusions}

In this paper, we used a dataset of 1,570,301 panelists of an opt-in online panel from eight counties from Europe, Latin America and North America, to analyze if Millennials differ in terms of survey participation from other age cohorts. We compared across generations the average participation and break-off rates, the average proportion of surveys answered with smartphones and the proportion of respondents who rated on average the surveys they answered with 4 or more stars.

\subsection{Main results and practical implications}

From our results, we can conclude that Millennials do differ from older cohorts in terms of survey participation, even if there are fewer differences than expected. Overall, we 
found that on average, Millennials have a significantly higher annual participation rate than older cohorts, confirming our Hypothesis 1. Thus, new strategies to engage and incentivize this generation are needed. However, Millennials have higher break-offs rates than Boomers only in half of the countries, while Generation X tends to abandon surveys in a higher extent than Millennials. Moreover, the differences in terms of break-off are very small, providing little support for our second hypothesis. Next, Millennials on average answered a significantly higher proportion of surveys using smartphones than older cohorts in all countries, which confirms our Hypothesis 3. As expected, the sizes of the differences are higher when compared with Boomers than with Generation X. Hence, practitioners must consider that if Millennials are part of their population of interest, they will answer most of the surveys using smartphones instead of computers. Thus, if surveys are not adapted to mobile devices, data quality and survey experience for this cohort might be negatively affected. Finally, no clear pattern has been found regarding survey evaluation. Considering these mixed results and the small size of the effects, we conclude that our Hypothesis 4 cannot be supported.

\subsection{Limits and further research}

However, these results have limits. First, although we used a very large dataset from 8 different countries, data comes from a unique online opt-in panel. Thus, future research could investigate if similar results hold for other online opt-in panels and countries, as well as for probability-based online panels.

Considering the higher proportion of surveys answered with smartphones by Millennials, future research should study how data quality and substantive conclusions may vary across cohorts if surveys are not adapted to smartphones. In addition, the high proportion of surveys that Millennials answer with smartphones open new opportunities for research. Smartphones have sensors as GPS, cameras or NFC that enhance the possibilities of collecting new forms of data (Wenz et al., 2017), as users' location, biomarkers or images.

Finally, strategies to improve the survey participation of Millennials could be tested. Previous research investigated how to improve respondents' motivation to participate for general population, for instance by using targeted survey designs (Lynn, 2017). Adapting these strategies to Millennials could improve their participation. In addition, making the survey experience more natural for Millennials may increase the engagement and future 
participation of this generation. For instance, allowing respondents to answer survey questions with images (Bosch et al., 2018).

\section{References}

Andreadis I (2015) Comparison of Response Times between Desktop and Smartphone Users. In: Mobile Research Methods: Opportunities and challenges of mobile research methodologies, pp. 63-79. Available from: http://www.ubiquitypress.com/site/chapters/10.5334/bar.e/.

Baker R, Blumberg SJ, Brick JM, et al. (2010) Research Synthesis: AAPOR Report on Online Panels. Public Opinion Quarterly, Oxford University Press 74(4): 711-781. Available from: https://academic.oup.com/poq/articlelookup/doi/10.1093/poq/nfq048 (accessed 25 June 2018).

Bosch OJ, Revilla M and Paura E (2018) Answering mobile surveys with images: an exploration using a computer vision API. Social Science Computer Review.

Bowen JT and Chen McCain SL (2015) Transitioning loyalty programs: A commentary on "the relationship between customer loyalty and customer satisfaction". International Journal of Contemporary Hospitality Management 27(3): 415-430.

Buskirk TD and Andrus CH (2014) Making Mobile Browser Surveys Smarter: Results from a Randomized Experiment Comparing Online Surveys Completed via Computer or Smartphone. Field Methods 26(4): 322-342.

Canada M (2015) Attention Spans. Available from: https://es.scribd.com/document/265348695/Microsoft-Attention-Spans-ResearchReport.

Gehan DS and Yamamoto O (2017) Relationship Between Millennials and Brand Loyalty: Mediating Brand Loyalty Factors. Journal of Business Theory and Practice 5(3): 223. Available from: http://www.scholink.org/ojs/index.php/jbtp/article/view/1042.

Hartman JL and McCambridge J (2011) Optimizing millennials' communication styles. Business Communication Quarterly 74(1): 22-44.

Kim D and Ammeter AP (2008) Examining shifts in online purchasing behaviors: decoding the 'net generation'. In: Academy of Information and ..., pp. 7-13. 
Available from:

https://www.alliedacademies.org/Public/Proceedings/Proceedings22/AIMS Proceedings.pdf\#page $=10$.

Lugtig PJ and Toepoel V (2016) Mobile-Only Web Survey Respondents. Survey Practice 9(3): 1-8. Available from:

https://dspace.library.uu.nl/handle/1874/339407 (accessed 10 May 2018).

Lynn P (2017) From standardised to targeted survey procedures for tackling nonresponse and attrition. Survey Research Methods 11(1): 93-103. Available from: https://ojs.ub.uni-konstanz.de/srm/article/view/6734 (accessed 25 June 2018).

Mavletova A and Couper MP (2013) Sensitive topics in PC web and mobile web surveys: Is there a difference? Survey Research Methods 7(3): 191-205. Available from: https://www.scopus.com/inward/record.uri?eid=2-s2.084890058064\&partnerID=40\&md5=8cc24a4796e96c8903143329788c26db.

Mayock P (2014) Personalization equals loyalty for millennials. Hotel News Now. Available from: http://www.hotelnewsnow.com/article/13067/Personalizationequals-loyalty-for-millennials.

Myers KK and Sadaghiani K (2010) Millennials in the workplace: A communication perspective on millennials' organizational relationships and performance. Journal of Business and Psychology 25(2): 225-238.

Pew Research Center (2014) Millennials in adulthood. Available from: https://archive.org/details/140307PewMillennialsinadulthood.

Revilla M (2017) Analyzing the survey characteristics,. participation,. and evaluation across 186 surveys in an online opt-in panel in Spain. Methods, data, analyses 11(2): 135-162.

Revilla M, Toninelli D, Ochoa C, et al. (2016) Do online access panels need to adapt surveys for mobile devices? Internet Research 26(5): 1209-1227.

Stein J and Sanburn J (2013) Why Millennials will save us all. Time International 181(19): 26-33.

Strauss W and Howe N (1991) Generations: The history of America's future, 1584 to 2069. New York, NY: William Morrow \& Company.

Wenz A, Jäckle A and Couper MP (2017) Willingness to use mobile technologies for 
data collection in a probability household panel. Understanding Society.

Williams KC and Page RA (2011) Marketing to the Generations. Journal of Behavioral Studies in Business 5: 1-17. Available from:

http://www.www.aabri.com/manuscripts/10575.pdf. 